\title{
Water Ice Formation and the o/p Ratio
}

\author{
François Dulieu \\ LERMA, UMR 8112 du CNRS \\ Observatoire de Paris et Université de Cergy Pontoise \\ 5, mail Gay Lussac, 95000 Cergy Pontoise, France \\ email: francois.dulieu@obspm.fr
}

\begin{abstract}
Water is observed in many astrophysical environments in both gas and solid phase. Water ice, for its specific properties, is probably the most important template that structures the gas-solid interaction. In cold environments, its synthesis is supposed to occur directly in the solid phase and then water acts as a catalytic matrix for subsequent synthesis of various molecules. When the medium begins to warm again, water sublimates and nourishes the gas phase, as occurs for example in comets or in star forming regions. Over the last four years, water formation on cold surfaces has been studied experimentally. Different precursors $\left(\mathrm{O}, \mathrm{O}_{2}, \mathrm{O}_{3} \ldots\right)$ have been used to understand the complex mechanisms that take place. Although numerous questions remain unanswered, at present, it is clear that water is easily formed by different pathways, and that the ice formed has an amorphous structure. The recent observations of the ortho/para ratio of water with Herschel satellite have similarities with the previous $\mathrm{o} / \mathrm{p}$ ratio observations of water in comets. Some experimental work have been recently reported in this domain, mostly rare gas matrix studies where nuclear spin conversion is measured even at $4.2 \mathrm{~K} . \mathrm{H}_{2}$ molecules adsorbed on amorphous solid water ice also exhibit a nuclear spin conversion in presence of a tiny fraction of $\mathrm{O}_{2}$. Finally, I will discuss if microphysics properties of water desorption can explain the $\mathrm{o} / \mathrm{p}$ ratio values observed.
\end{abstract}

Keywords. astrochemistry, molecular processes, methods: laboratory, ISM: molecules

\section{Introduction}

Hydrogen and oxygen are the two most abundant reactive atoms. $\mathrm{H}_{2}$ is the most abundant molecule in the universe and is mainly present in the gas phase, even if the solid phase chemistry is thought to be crucial for its formation. Also, adsorbed $\mathrm{H}_{2}$ may play an important role in the deuteration of species (Kristensen et al. (2011)).

Water is the most abundant molecule in the solid phase. It has been detected in gaseous or solid form in numerous astrophysical environments such as planets, comets, interstellar clouds and star forming regions (Ehrenfreund et al. (2003), Dartois (2005), Tielens (2005)). Amorphous water ice was directly detected in dark interstellar clouds through infra-red absorption bands Leger et al. (1979).

Water has the property to create H-bonds, and this has several kinds of consequences. The first consequence is that solid water ice has a high energy of sublimation (46.9 kJ per mole) (Speedy et al. (1996), Fraser et al. (2001)). This is why this molecule is often in its solid form, because the range of conditions where solid water ice can exist is wider than for the majority of other small molecules detected in space. The second main implication is that water shapes the morphology and geometry of the solid phase because it is the main compound of icy mantles and has rather strong interactions with the adsorbates. The morphology of the solid phase has important effect on gas-grain interactions, i.e, by changing the binding properties of adsorbed molecules (Fillion et al. (2009)), by changing the kinetics of reactions like in the case of $\mathrm{H}_{2}$ formation (Hornekaer 
et al. (2003), Congiu et al. (2009)). Moreover, the gas-grain interaction is only the first step of molecular synthesis that occurs in solid phase. In addition, surface chemistry is of course influenced by the morphology of the ice, for example in the case of D diffusion in function of the thickness of the porous film (Matar et al. (2008)), or in the case of hydrogenation of CO (Hidaka et al. (2007)).

There is a wealth of models that predict that water should be synthesized on the surface of dust grains (e.g., Cuppen \& Herbst (2007) and references therein). The first part of the present paper focuses mainly on experimental studies of the water formation itself on cold solid substrates. The neutral gas phase route is known have a low efficiency because the $\mathrm{OH}+\mathrm{H} \longrightarrow \mathrm{H}_{2} \mathrm{O}$ has an activation barrier. The main difference between the gas phase and the solid phase is that typical collision timescale in gas phase can be a day, whereas in solid phase, if the reactants are nearby, the frequency of attempts is typically of the order of the picosecond. Therefore, the main question in solid state is "Are the reactants nearby?" rather than "What is the height of the activation barrier of the reaction?". The diffusion properties, densities on the surface and the residence time compared to accretion time are probably of higher importance than the height of activation barriers. If the reaction can proceed in a time shorter than the timescale of the change in the environmental configuration of either reactant, the reaction occurs, no matter how short this time may be (picosecond, nanosecond, millisecond...).

The varieties of the surface conditions (surface densities of the reactants, temperature, fluxes, composition and morphology) studied in the recent experimental reports tend to make the comparison between different experimental results very hard and of difficult interpretation for the non-experimental astrochemists. The aim of the first part of this article is to show that some important and apparent trends can be drawn. One of them is that the water (and hydrogen peroxide) can be easily formed from any of the oxygen precursor deposited on a cold (10-20 K) substrate exposed to $\mathrm{H}$ atoms, and the that first step of hydrogenation is barrierless. The second important fact is that the water formed by surface reactions is amorphous and compact.

The second part of this article is devoted to the question of ortho-to-para ratio of molecules in connection with the solid state. Molecules having two protons that can be exchanged like in $\mathrm{H}_{2}, \mathrm{H}_{2} \mathrm{O}, \mathrm{H}_{2} \mathrm{CO}$.. have different spin isomers. The change of one isomer to the other requires a flip of nuclear spin. The nuclear spin conversion (NSC) may happen if interactions, such as for example strong magnetic gradients, are present. Nuclear spin conversions are also favored if the isomer states are energetically close. For a molecule in gas phase, it is believed that the nuclear spin conversion is not efficient because the interaction (collision) time between two molecules is too short. Of course, for the total population of the molecules, nuclear spin conversion can happen especially in the case where molecules are formed and destroyed. Accretion to solid phase and successive desorption to gas phase are processes that can drive, at least partly, the orthoto-para ratio observed in different astrophysical environments. In the case of comets, where desorption plays an obvious role, the opr of water has been found to be between 1.8 and 3, with a maximum around 2.4, corresponding spin temperature of around $30 \mathrm{~K}$ (Bonev et al. (2007), Bockelée-Morvan et al. (2009)). This value does not correspond to the kinetic temperature, and it is also not the case for $\mathrm{H}_{2}$, in shock regions (Neufeld et al. (2006)).

The Herschel satellite provides new input for interstellar medium regions Lis et al. (2010), Melnick et al. (2010)) and also for $\mathrm{D}_{2} \mathrm{O}$ opr (Vastel et al. (2010)). The question of the origin of the observed diversity is nowadays examined as never before.

The aim of this paper is to present the recent progress made in the laboratory or theoretical field, and to try to see if the microphysics of the gas-grain interaction can 
have an effect on the opr ratio observed. I will first discuss the case of $\mathrm{H}_{2}$ adsorbed on the surface of solid water ice, and show that a tiny fraction of paramagnetic molecules such as $\mathrm{O}_{2}$ is able to produce NSC in a time measurable in the laboratory. I will present some results obtained in noble gas matrix that demonstrate the dependence of the NSC on the concentration of $\mathrm{H}_{2} \mathrm{O}$, suggesting that the NSC is accelerated with water-water interaction. I will recall the theoretical results obtained by Buntkowsky et al. (2008), that show that the interaction of 2 water molecules should produce NSC in the millisecond timescale. I will finally discuss the possibility that selective desorption of water in function of the spin isomer can produce a disequilibrium in the opr of the nascent molecules detected in the gas phase.

\section{Experimental studies of water formation}

During the last 5 years, water formation on cold surfaces has been studied experimentally. In 2006 our group presented the first successful attempt to synthesize water molecules on a cold substrate starting from oxygen and hydrogen. This experiment was carried out on a water substrate held at $10 \mathrm{~K}$, mimicking thus the growth of water mantles (Dulieu et al. (2006)). However, the chosen system was by far the most complicated to study even if the qualitative results were clear, namely, production of water. (Dulieu et al. (2010)). The first complication was provided by the water template, which, though perfectly relevant to astrophysical purposes, increased greatly the difficulty of the analysis. The water substrate makes the analysis by infrared spectroscopy very hard, and the isotope exchange $\left(\mathrm{H}_{2} \mathrm{O}+\mathrm{D}_{2} \mathrm{O} \rightleftharpoons 2\right.$ HDO) occurring during the desorption of water makes the Temperature Programmed Desorption (TPD) analysis much more complex. The second intrinsic difficulty is that oxygen atoms were used as reactants together with $\mathrm{D}$ atoms. Under $\mathrm{O}$ and $\mathrm{H}$ irradiation of a cold surface, $\mathrm{O}$ atoms can as well react with themselves so the $\mathrm{O}+\mathrm{H}, \mathrm{O}_{2}+\mathrm{H}$ and $\mathrm{O}_{3}+\mathrm{H}$ pathways are simultaneously open. Therefore, the reactions network leading to the observed production of water is of maximal complexity. The first column of Table 1 , presents 16 of the 18 reactions proposed as active in the formation of water in solid phase, starting from pure $\mathrm{O}$ or $\mathrm{H}$ species. Beside the complexity of this first attempt, the conclusions of this study are that oxygen atoms are included efficiently in the water formed, although it was not possible to determine unambiguously which chemical route led to water and its exact efficiency.

One claim of this work was that the $\mathrm{O}_{2}+\mathrm{H}$ reaction was very efficient and it has been rapidly explicitly demonstrated by two independent group in Japan (Miyauchi et al. (2008)) and in the Netherlands (Ioppolo et al. (2008)). In contrast with the previous study, the most abundant compound formed was not water, but $\mathrm{H}_{2} \mathrm{O}_{2}$. In these two studies a thick $\mathrm{O}_{2}$ layer was exposed to $\mathrm{H}$ (or D) atoms and the water formation was identified following the formation of hydrogen peroxide. Later, in other experiments where several $\mathrm{H} / \mathrm{O}_{2}$ ratios were investigated, water was found to become the major product as soon as the $\mathrm{H} / \mathrm{O}_{2}$ became very large (Oba et al. (2009)).

Ozone too was been used as a precursor at submonolayers coverages on a water substrate on which $\mathrm{O}_{3}$, exposed to $\mathrm{H}$, mostly leads to the formation of water (Mokrane et al. (2009)). In the case of $\mathrm{a} \mathrm{O}_{3}$ ice used as a substrate, water and also hydrogen peroxide are formed (Romanzin et al. (2011)). As a last part of a complex experimental puzzle, infrared spectroscopy allowed to isolate some reaction intermediates such as $\mathrm{O}_{2} \mathrm{H}$ and $\mathrm{OH}$, and to propose a complex chemical network (Ioppolo et al. (2010), Cuppen et al. (2010)).

One common result of all these studies is that water is always produced starting from $\mathrm{O}, \mathrm{O}_{2}$ or $\mathrm{O}_{3}$ by addition of $\mathrm{H}$ atoms, regardless of the substrate. However, some obvious and important discrepancies appear such the $\mathrm{H}_{2} \mathrm{O}_{2} / \mathrm{H}_{2} \mathrm{O}$ ratio. These discrepancies are 
Table 1. Proposed reactions for water formation on cold solid state from oxygen and hydrogen. Opinion of (o) H. Linnartz (Leiden Observatory ), (*) N. Watanabe (Okkaido University) and $(\dagger)$ F. Dulieu (Paris Observatory). ***: Certain, ** : Presumption, * : Opinion

\begin{tabular}{|c|c|c|c|c|}
\hline Reaction & \# & No Barrier & Barrier & No reaction \\
\hline $\mid \begin{array}{l}\mathrm{O}+\mathrm{O} \longrightarrow \mathrm{O}_{2} \\
\mathrm{O}_{2}+\mathrm{O} \longrightarrow \mathrm{O}_{3}\end{array}$ & $\left|\begin{array}{l}1 \\
2\end{array}\right|$ & $\begin{array}{l}* * \dagger \dagger \\
* * *\end{array}$ & $\dagger \dagger \circ$ & \\
\hline $\begin{array}{l}\mathrm{O}+\mathrm{H} \longrightarrow \mathrm{OH} \\
\mathrm{O}_{2}+\mathrm{H} \longrightarrow \mathrm{HO}_{2} \\
\mathrm{O}_{3}+\mathrm{H} \longrightarrow \mathrm{O}_{2}+\mathrm{OH}\end{array}$ & $\left|\begin{array}{l}3 \\
4 \\
5\end{array}\right|$ & $\begin{array}{l}* * \dagger \dagger \circ \\
* * * \dagger \dagger \text { ○०० } \\
* * \dagger \dagger \dagger \text { ○ }\end{array}$ & & \\
\hline $\begin{array}{l}\mathrm{HO}_{2}+\mathrm{H} \longrightarrow \mathrm{H}_{2} \mathrm{O}_{2} \\
\mathrm{HO}_{2}+\mathrm{H} \longrightarrow \mathrm{OH}+\mathrm{OH} \\
\mathrm{HO}_{2}+\mathrm{H} \longrightarrow \mathrm{H}_{2} \mathrm{O}+\mathrm{O}\end{array}$ & $\left|\begin{array}{l}6 \\
7 \\
8\end{array}\right|$ & $\begin{array}{l}* * * \dagger \circ \circ \circ \\
* \dagger \circ \circ \circ \\
\dagger \circ \circ \circ\end{array}$ & & \\
\hline $\begin{array}{l}\mathrm{OH}+\mathrm{H} \longrightarrow \mathrm{H}_{2} \mathrm{O} \\
\mathrm{OH}+\mathrm{OH} \longrightarrow \mathrm{H}_{2} \mathrm{O}_{2} \\
\mathrm{OH}+\mathrm{OH} \longrightarrow \mathrm{H}_{2} \mathrm{O}+\mathrm{O}\end{array}$ & $\left|\begin{array}{c|}9 \\
12 \\
13\end{array}\right|$ & $\begin{array}{l}* * \dagger \dagger \circ 0 \\
* * * \dagger \circ \circ \circ \\
* * * \dagger\end{array}$ & $\dagger$ & \\
\hline \multicolumn{3}{|c|}{$\left|\mathrm{H}_{2} \mathrm{O}_{2}+\mathrm{H} \longrightarrow \mathrm{H}_{2} \mathrm{O}+\mathrm{OH}\right| 14 \mid$} & \multicolumn{2}{|l|}{$|* * * \dagger \dagger \circ ০ \circ|$} \\
\hline $\begin{array}{l}\mathrm{O}+\mathrm{H}_{2} \longrightarrow \mathrm{H}_{2} \mathrm{O} \\
\mathrm{O}_{2}+\mathrm{H}_{2} \longrightarrow \mathrm{H}_{2} \mathrm{O}+\mathrm{OH} \\
\mathrm{OH}+\mathrm{H}_{2} \longrightarrow \mathrm{H}_{2} \mathrm{O}+\mathrm{H} \\
\mathrm{O}_{3}+\mathrm{H}_{2} \longrightarrow \mathrm{H}_{2} \mathrm{O}+\mathrm{O}_{2}\end{array}$ & $\begin{array}{l}15 \\
16 \\
17 \\
18\end{array}$ & & $* * \circ \circ$ & $\begin{array}{c}* * * \dagger \dagger \\
* * * \dagger \dagger \dagger 00 \circ \\
\dagger \dagger \\
\dagger \dagger \dagger \text { ○०० }\end{array}$ \\
\hline
\end{tabular}

probably due to different experimental conditions, and most of all the accessibility to the reactants (surface density, mobility, diffusion...) which is of major importance as explained earlier.

In order to show that the experimental results present more similarities than differences, I asked the two leaders of the other group their opinion about the chemical reactions that occur on a cold surface at $10 \mathrm{~K}$. Table 1 presents 16 reactions that can occur on a cold substrate in the water formation network. The reactions may have no noticeable barrier and appear to be very efficient. It is for example the case of $\mathrm{O}_{3}+\mathrm{H}$ which occurs even at $50 \mathrm{~K}$, a temperature at which the residence time of $\mathrm{H}$ on the substrate is extremely short. Reactions can have a barrier and therefore, some competing mechanism can prevent the reaction to occur or tend to minimize its role, or a certain product may take more time to develop with respect to others. No reaction, in Table 1 , means that under these experimental conditions it is not possible to detect any effect such as the case of $\mathrm{O}_{2}+\mathrm{H}_{2}$.

Table 1 shows that there is a large consensus on most of the reactions. For those reactions involving only oxygen (\#1 and \#2) there is still a question about the presence or not of an activation barrier in the formation of ozone by addition of $\mathrm{O}$ and $\mathrm{O}_{2}$. On the contrary, it is clear that $\mathrm{O}, \mathrm{O}_{2}$ and $\mathrm{O}_{3}$ undergo hydrogenation very efficiently.

The two reaction intermediates $\mathrm{OH}$ and $\mathrm{O}_{2} \mathrm{H}$ promptly react with $\mathrm{H}$, but also together, and basically will form $\mathrm{H}_{2} \mathrm{O}$ or $\mathrm{H}_{2} \mathrm{O}_{2}$ at the end of the chain, but the branching ratios are still an open question.

$\mathrm{H}_{2} \mathrm{O}_{2}$ can react with $\mathrm{H}$, but this reaction is slower than any oxygen-hydrogen reaction (typically one order of magnitude slower). This can be attributed to a barrier. However, as $\mathrm{H}_{2} \mathrm{O}_{2}$ is thought to react only with $\mathrm{H}$, whenever it is accessible to $\mathrm{H}$ atoms, it will end up in water formation. This is mainly the reason why water appears always to be 
the major final compound in case of sub-monolayer reactions, that is when complete hydrogenation can be achieved.

Finally $\mathrm{H}_{2}$, which is always present in our experimental conditions, as well as on icy dust grains in dark cloud, does not participate in the water formation except maybe for the $\mathrm{OH}+\mathrm{H}_{2}$ reaction. Yet, our group has never succeeded to demonstrate this possible reaction in a direct way. It is, however, one of the key reaction that should regulate the deuterium enrichment of water (Tielens et al. (1983), Kristensen et al. (2011)).

Another important aspect of the experimental study of water formation is that the morphology of the ice produced has been determined to be compact (Oba et al. (2009), Accolla thesis (2010)), and even if porosity can arise from the adsorption of gaseous water on cold dust grains, the molecular hydrogen formation is able to reduce dramatically the porosity of the ice. This is likely to be due to the fraction of the formation energy released which acts as a local heating of the ice network, and thus reduces the porous nature of water ice (Accolla et al. (2011)).

The experimental study of water formation has been extensively studied over the last 5 years, and has demonstrated that the water formation on dust grains is plausible. Thanks to the variety of precursors and of experimental conditions, the chemical networks involved have been determined even if some open questions still remain.

There are also some challenging matters like the determination of the fraction of compounds that could desorb directly during the formation phase, due to the large energy released. The study of the first layer of water on silicates (see poster by Vidali et al.) or carbonaceous material has to be investigated and probably the fraction of ejected molecules upon formation is even more important in such a context.

\section{Nuclear spin conversion on cold surfaces}

Because their protons have two interchangeable identical positions, molecules like $\mathrm{H}_{2}$, $\mathrm{H}_{2} \mathrm{O}$ or $\mathrm{H}_{2} \mathrm{CO}$ have spin isomers. The spin isomers are sometimes called by extension ortho and para molecules, according to their nuclear spin states. The total wave function of such molecules shall be antisymmetric, so for a given nuclear spin parity exists a rovibrational wave function of opposite parity. For the case of $\mathrm{H}_{2}$, in the $v=0$ state, the odd $J$ rotational numbers correspond to ortho population $(\mathrm{I}=1$, triplet) and the even $J$ states correspond to the para population ( $\mathrm{I}=0$, singlet).

If such a molecule is perfectly isolated and we consider that the nuclear spin has no interaction with the rest of the molecule, the nuclear spin cannot flip and therefore the conversion between the two spin isomers is not possible. This is why one can consider this spin isomers as two independent population of molecules.

One of the new capabilities opened by the Herschel satellite is to be able to measure, in some environments, the ortho-to-para (opr) ratio of water (e.g. Lis et al. (2010)). In different astrophysical context, especially in comets, it is already well established that the ratio between the two spin isomer populations can define a spin temperature that does not correspond to the kinetic temperature (Croviser (1998)). At high temperature $(\mathrm{T}>300 \mathrm{~K})$ the opr is equal to the statistical weight of the nuclear spin degeneracy $\simeq 3$ ( $3 / 4$ for ortho molecules and $1 / 4$ for para molecules).

Let us consider the case of $\mathrm{H}_{2}$. Figure 1 shows the distribution of the population considering two extreme cases of no nuclear spin conversion, hence two separate populations (right panel) and a fast spin conversion which allows the two spin isomers to be considered as a unique population (central panel). The two cases are presented for two different temperatures. At $30 \mathrm{~K}$ (and below) the molecules are in the lowest possible rotational states. If conversion is possible, all the molecules are in the $J=0$ state. In the case of two 


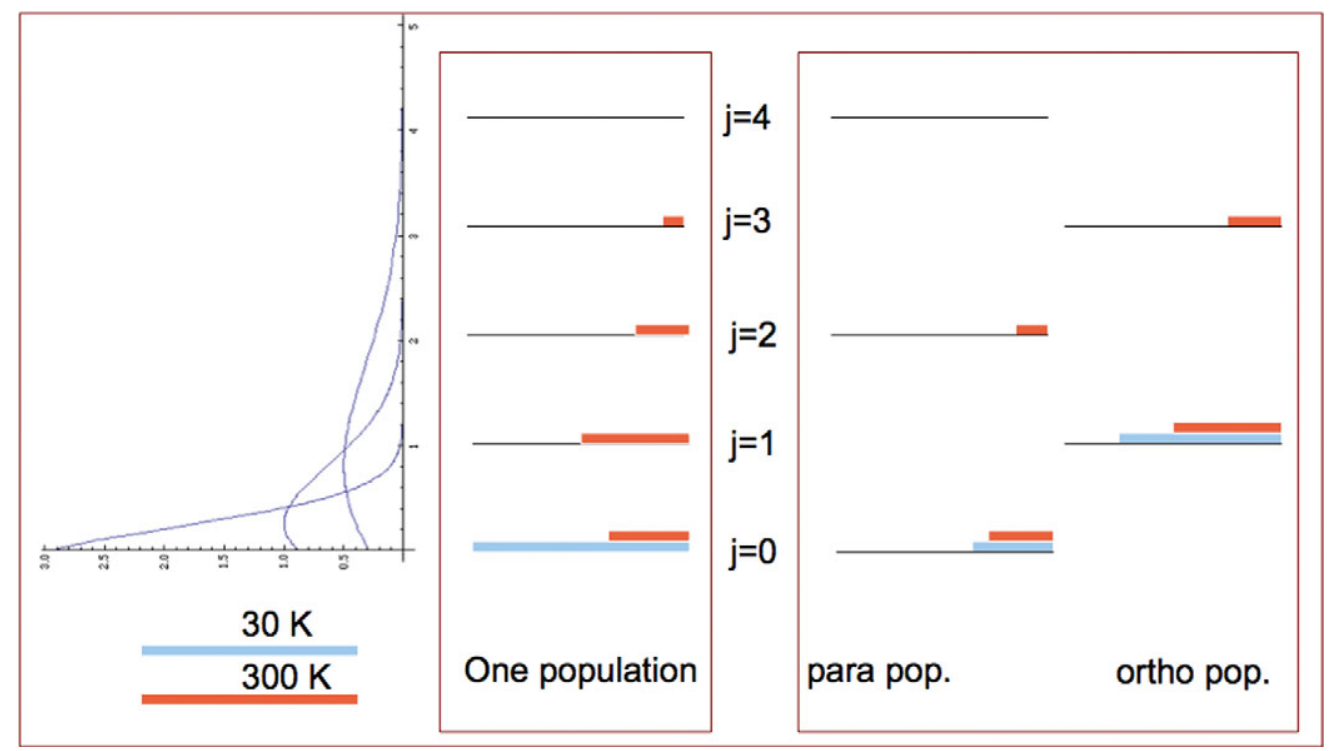

Figure 1. Relative population of $\mathrm{H}_{2}$ molecules for a gas at $30 \mathrm{~K}$ (blue bars), and $300 \mathrm{~K}$ (orange bars) of the $v=0$ state in function of the $J$ rotational states. The left panel represents the normalized continuous Boltzmann distribution (at $30 \mathrm{~K}, 100 \mathrm{~K}$ and $300 \mathrm{~K}$ ) as a guide for the eye. The central panel represents the case of a unique spin isomer population (obtained if NSC is very efficient), and the right panel corresponds to two different isomer populations populated with a opr $=3$.

separate populations, $1 / 4$ of the molecules is in the $J=0$ state, and $3 / 4$ is in the $J=1$ state which is the lowest state of para molecules. At $300 \mathrm{~K}$, the molecules are spread over the 4 first levels. Under either assumption on nuclear spin conversion (NSC), opr is equal to 3 . In the case of a unique population, we can notice that at very low temperature opr $=0$, and when the temperature increases the opr grows to reach the asymptotic value of 3 at $\mathrm{T}>250 \mathrm{~K}$. If the opr is comprised in the $[0,3]$ range, it corresponds to a spin temperature. In numerous astrophysical observations, this spin temperature does not correspond to the kinetic temperature.

On water ice surfaces, $\mathrm{H}_{2}$ desorbs below $30 \mathrm{~K}$ (Amiaud et al. (2006) Fillion et al. (2009)). Let's then consider the simple experiment consisting in adsorbing $\mathrm{H}_{2}$ at $10 \mathrm{~K}$ and heating the water ice sample until $\mathrm{H}_{2}$ desorbs (the so called Temperature Programmed Desorption). If nuclear spin conversion happens very rapidly at $10 \mathrm{~K}$, all the molecules should desorb in the $J=0$ rotational state. If nuclear spin conversion does not occur, therefore $3 / 4$ of the molecules should desorb in the $J=1$ state and $1 / 4$ in the $J=0$. Using a tunable laser, it is possible to detect selectively a quantum state (Selected Quantum State TPD). In the case of $\mathrm{H}_{2}$ (or $\mathrm{D}_{2}$ ) it is clear that the second scenario, i.e. no NSC, corresponds to the experiments. We have measured that molecules at $300 \mathrm{~K}$ (having an opr $=3$ ), deposited at $10 \mathrm{~K}$, desorb below $30 \mathrm{~K}$ and have a population of both $J=0$ and $J=1$ states.

We have also noticed that the dynamic of the desorption is slightly different because of an energy difference in the adsorption energy of ortho and para states (Amiaud et al. (2008)). For $\mathrm{D}_{2}$ this difference has been estimated to be $1.4 \pm 0.3 \mathrm{meV}$. We must note that the $J=1$ molecules are more bound to the surface than the $J=0$ ones, which is a little counterintuitive, because one could think that rotation may favor the desorption process. This underlines the fact that the $\mathrm{D}_{2}$ molecules can rotate almost freely on the 
water ice substrate. The case of $\mathrm{H}_{2}$ adsorbed on a water surface is very similar to a gas phase problem, in the sense that the ro-vibrational wave function is clearly defined and therefore the spin isomers keep fully their meaning. The great difference with the gas phase is that the time of interaction with the local surroundings can be very long.

We have, therefore, performed another set of experiments, basically the same as above, but where we varied the time (from one minute to one hour) between the adsorption and the desorption phase for $\mathrm{H}_{2}$ and $\mathrm{D}_{2}$ molecules to see if some conversion happens during the wait time. We were able to detect a NSC in the case where we added $\mathrm{O}_{2}$ molecules on the substrate (Chehrouri et al. (2011)). We observed a typical NSC time of one hour in the case where $3 / 10000$ adsorption sites where occupied by $\mathrm{O}_{2}$ molecules and an occupation of $>1 / 6$ of $\mathrm{D}_{2}$ molecules. The NSC efficiency increased with the $\mathrm{O}_{2}$ coverage and with the $\mathrm{D}_{2}$ coverage. The conclusion of this study is that even trace quantities of $\mathrm{O}_{2}$ (or other paramagnetic molecules) present on the surface is able to induce NSC at the astrophysical time scale.

Infra red spectroscopy allows to detect ortho and para states of water imbedded in a rare gas matrix. Even at 4.2 K, NSC occurs (Pardanaud et al. (2008)), but in this case with a typical timescale of one day. Particularly, the NSC increases with the concentration of water molecules demonstrating that even at long range, water-water interaction is sufficient to induce the conversion. Once again, the case of rare gas matrix is not exactly the case of water in solid phase, because the water molecule in the rare gas cage can rotate with small constraints. The nature of the rare gas case affects the NSC timescale (Pardanaud thesis (2007)). The ortho and para definition of molecules in these systems is therefore close to the one in gas phase.

But in solid phase, which means in the case of water in solid ice, water molecules form $\mathrm{H}$ bonds, and the rotation is widely impeded. Buntkowsky et al. (2008) have studied the case of two water molecules from a theoretical point of view. They have shown that the frustration of the rotation reduces considerably the difference in energy of the rotational levels. This implies that NSC in water ice should happen in the millisecond range.

Considering that the NSC on solid water ice is accelerated by the presence of paramagnetic species, or simply by the frustration of rotation, it is reasonable to think that for one molecule the NSC is faster that the desorption process in an astrophysical context. Figure 2 represents a schematic view of water desorption taking into account the ortho and para water molecules.

We have proposed a simple model of water desorption that includes the possibility of spin conversion in solid phase, and takes into account a differential desorption of the ortho and para molecules and no efficient spin conversion in the gas phase Dulieu et al. in prep. We assume a rapid (compared to desorption) NSC in the solid phase, hence one population can be transferred to another, if one of the two populations evaporates more efficiently. If we consider that the rotational energy difference in the gas phase induces differences in the desorption energy, therefore one can see that the para molecules (which include the 000 state) have on average a lower desorption energy and as a consequence a higher desorption flux. We have estimated that in the 100-120 K range, the opr $\sim 2.4$ just after desorption. Para molecules have a higher desorption probability, and that their reduction in number (due to the desorption) is compensated by rapid NSC in solid phase. This is why the selective desorption favors the para population and reduce apparently the spin temperature. The conclusion is that the desorption process could be one of the reason why the observed spin temperature does not correspond to the kinetic temperature. New experiments are required to validate this proposition. 


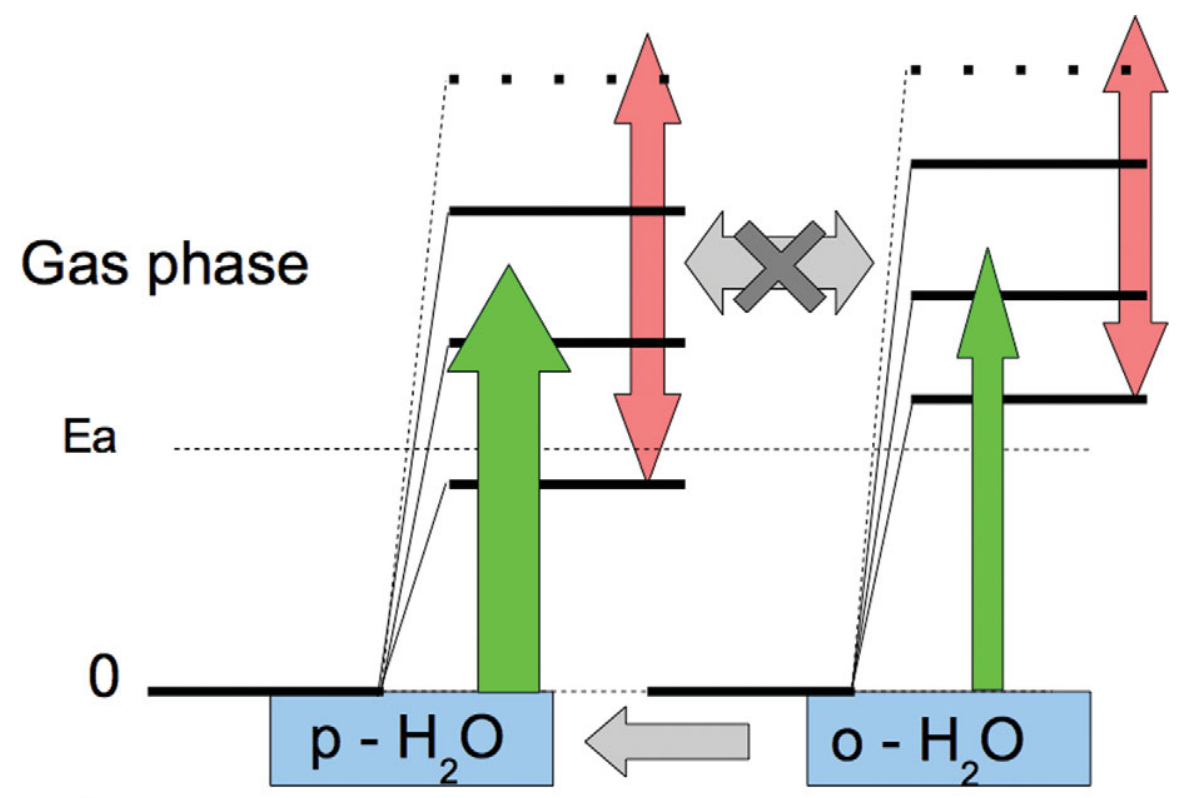

\section{Solid phase}

Figure 2. Schematic representation of the model of selective desorption. Large gray arrow: Nuclear Spin conversion; Green arrows: desorption; Salmon arrow: thermal equilibrium induced by gas phase collisions.

\section{Conclusion}

Numerous experimental studies during the last years have demonstrated that the water formation on cold solid surface is a efficient mechanism, mainly because no activation barrier has been measured for the first step of hydrogenation of any pure oxygen molecules $\left(\mathrm{O}, \mathrm{O}_{2}, \mathrm{O}_{3}\right)$. The reaction network is, however, complex but a rather good accord exists in the community. Nevertheless, some very interesting questions should be addressed in the next years. Among these questions, the fraction of water molecules that can directly go into the gas phase upon their formation will be probably one of the most stimulating.

Also the ortho and para topic in solid state has made some interesting progress, especially for the $\mathrm{H}_{2} / \mathrm{H}_{2} \mathrm{O}$ system and for rare gas matrix experiments. There is for the moment no experimental evidence of how the spin isomers evolve when the solid-gas phase transition occurs. However, we have proposed a simple explanation why spin temperature and kinetic temperature should not be the same after a simple thermal desorption.

\section{Acknowledgement}

I would like to thanks Dr Michaut and Pr Fillion (Université Pierre et Marie Curie), Pr Linnartz (Leiden Observatory) and Pr Watanabe (Okkaido University) for our exchanges before the conference, and Dr Congiu for his help after the conference.

\section{References}

Mario Accolla PHD thesis, 2010.

Accolla, M., Congiu, E., Dulieu, F., Manicò, G., Chaabouni, H., Matar, E., Mokrane, H., Lemaire, J. L., \& Pirronello, V., 2011, Phys. Chem. Chem. Phys., 13 (17), 8037 - 8045 
Amiaud, L., Fillion, J. H., Baouche, S., Dulieu, F., Momeni, A., \& Lemaire, J. L. 2006, J. Chem. Phys., 124, 094702

Amiaud, L., Momeni, A., Dulieu, F., Fillion, J. H., Matar, E., \& Lemaire, J. L., 2008, Phys. Rev. Lett., 100, 056101.

Bockelée-Morvan D. Woodward, C. E., Kelley, M. S., \& Wooden D. H., 2009, ApJ 696, 2737

Bonev B. P., Mumma, M. J., Villanueva, G. L., Disanti M. A. et al., 2007, ApJ, 661, L97

Buntkowsky G., Limbach H.-H., Walaszek B., Adamczyk A., Xu Y., Breitzke H., Schweitzer A., Gutmann T., Warchtler M., Frydel J., Emmler Th., Amadeu N., Tietze D., Chaudret B., 2008, Z. Phys. Chem. 22210491063

Cherhouri M., Fillion J-H, Chaabouni, H. and Mokrane et al.Phys. Chem. Chem. Phys., 13, 2172

Congiu E., Matar E., Kristensen L. E., Dulieu F. and Lemaire J. L., 2009, Mon. Not. R. Astron. Soc. 397, L96

Cuppen, H. M. \& Herbst, E. 2007, ApJ, 668, 294

Cuppen, H. M., Ioppolo, S., Romanzin, C., van Dishoeck, E. F., \& Linnartz, H, 2010, Phys. Chem. Chem. Phys., 12, 12077

Croviser, J., 1998, Faraday discuss. 109, 437.

Dartois, E. 2005, Space Science Reviews, 119, 293

Workshop: Molecular databases for Herschel, ALMA and SOFIA from 6-8 Dec 2006, Lorentz center, Leiden, The Netherlands.

Dulieu, F., Amiaud, L., Fillion, J.-H.., Matar, E., Momeni, A., Pirronello, V., \& Lemaire, J. L. 2007, in Molecules in Space and Laboratory, Ed. J.L. Lemaire, F. Combes., p. 79

Dulieu, F., Amiaud, L., Congiu, E., Fillion, J.-H., Matar, E., Momeni, A., Pirronello, V., \& Lemaire, J. L. 2010, A\&BA, 512, A30

Dulieu F., Congiu E., Fillion J.H., Michaut X. in preparation.

Ehrenfreund, P., Fraser, H. J., Blum, J., Cartwright, J. H. E., Garcìa-Ruiz, J. M., Hadamcik, E., Levasseur-Regourd, A. C., Price, S., Prodi, F., \& Sarkissian, A. 2003, Planetary and Space Science, 51, 473

Fraser, H. J., Collings, M. P., MacCoustra M. R. S., \& Williams, D. A., 2001, Mon. Not. R. Astron. Soc. 327,11651172

Fillion, J.-H., Amiaud, L., Congiu, E., et al. 2009, Phys. Chem. Chem. Phys., 11, 4396

Hidaka, H., Kouchi, A., \& Watanabe, N., 2007, J. Chem. Phys., 126, 204707

Hornekaer, L., Baurichter A ., Petrunin, V. V., Field. D. \& Luntz, A., C., 2003 Science, 302, 1943

Hiraoka, K., Miyagoshi, T., Takayama, T., Yamamoto, K., \& Kihara, Y. 1998, ApJ, 498, 710

Ioppolo, S., Cuppen, H. M., Romanzin, C., van Dishoeck, E. F., \& Linnartz, H. 2008, ApJ 686 1474

Ioppolo, S., Cuppen, H. M., Romanzin, C., van Dishoeck, E. F., \& Linnartz, H. 2010, Phys. Chem. Chem. Phys., 12, 12065

Kristensen, L. E., Amiaud, L., Fillion, J.-H., Dulieu, F., \& Lemaire, J.-L., 2011, A $\mathscr{\ddots} A$, 527, A44

Oba Y., Miyauchi N., Hidaka H., Chigai T., Watanabe N. and Kouchi A., Astrophys. J., 2009, 701,464 .

Lis D. C., Phillips, T. G., Goldsmith, F., \& Neufeld, D. A., et al. 2010, A\& A, 521, L26,

Leger, A., Klein, J., de Cheveigne, S., Guinet, C., Defourneau, D., \& Belin, M. 1979, A\&A 79, 256

Matar, E., Congiu, E., Dulieu, F., Momeni, A., \& Lemaire, J. L. , 2008, A\&\&A, 492, L17-L20

Mokrane H., Chaabouni H., Accolla M., Congiu E., Dulieu F., Chehrouri M., Lemaire J. L., 2009, ApJ ,705, L195

Melnick, G. J., Tolls, V., Neufeld D. A., Bergin E. A., et al., 2010, A\&\&A, 521, L27

Miyauchi, N., Hidaka, H., Chigai, T., Nagaoka, A., Watanabe, N., \& Kouchi, A. 2008, Chemical Physics Letters, 456, 27

Neufeld, D. A., Melnick G. J., Sonnentrucker, P., Bergin, E. A. et al., 2006, ApJ, 649, 816.

Cedric Pardanaud PHD thesis, 2007, http://tel.archives-ouvertes.fr/tel-00259961/fr/

Pardanaud, C., Vasserot, A.-M., Michaut, X., and Abouaf-Marguin, L., (2008), Journal of Molecular Structure 873, 181 
Romanzin, Ioppolo, S., C., Cuppen, H. M., van Dishoeck, E. F., \& Linnartz, H., 2011, J. Chem. Phys, 134, 084504

Speedy, R. J., Debenedetti, P. G., Scott Smith R., Huang, C., \& Kay, B. D., 1996, J. Chem. Phys. 105, 240.

Tielens, A. G. G. M., Hagen, W., \& Greenberg, J. M. 1983, J. Phys. Chem., 87, 4220

Tielens, A. G. G. M. 2005, The Physics and Chemistry of the Interstellar Medium (Cambridge University Press)

van Dishoeck, E. F. 2004, Annu. Rev. Astron. Astrophys., 42, 119

Vastel, C., Ceccarelli, C., Caux, E., Coutens, A., et al., 2010, A\& A, 521, L31

\section{Discussion}

\section{HornekAeR: Chairwoman}

ERIC HeRBst: Why does $\mathrm{H}_{2}$ o/p conversion require $\mathrm{O}_{2}$ whereas $\mathrm{H}_{2} \mathrm{O}$ does not?

Changing the concentration of $\mathrm{O}_{2}$ on the substrate is a way to control experimentally the $\mathrm{o} / \mathrm{p}$ conversion time of $\mathrm{H}_{2}$ and makes it measurable in an easier way under our experimental conditions. The $\mathrm{O}_{2}$ present on the substrate probably also affects the $\mathrm{o} / \mathrm{p}$ conversion of $\mathrm{H}_{2} \mathrm{O}$, but it has not been yet measured in ice. In absence of paramagnetic species, the difference between $\mathrm{H}_{2}$ and $\mathrm{H}_{2} \mathrm{O}$ adsorbed on the substrate is mostly due to the fact that $\mathrm{H}_{2}$ can rotate almost freely at the surface whereas water form $\mathrm{H}$ bonds and therefore has very important constraints. In one case the rotational energy is almost unchanged, and in the other case, the rotational energy levels are almost degenerated, and this strongly favors the NSC.

NAOKI Watanabe: Why does the tiny addition of $\mathrm{O}_{2}$ accelerate conversion of $\mathrm{H}_{2}$ ? For the ratio $\mathrm{H}_{2} / \mathrm{O}_{2} 10^{-5}$, it is quite rare to be close to $\mathrm{O}_{2}$ on the surface.

$\mathrm{O}_{2}$ offers a magnetic gradient that provokes NSC. The conversion is complete in the case of $\mathrm{D}_{2}$ which means that these molecules are mobile at $10 \mathrm{~K}$ and are able to adsorb (at least temporarily) close to one $\mathrm{O}_{2}$ molecule. If molecules were not mobile only very little conversion would occur.

NAOKI WATANABE: Have you measured the surface-temperature dependence of o/p conversion of $\mathrm{H}_{2}$ ?

No we haven't, but it is an excellent idea, not necessary easy to realize. If we increase the temperature, the adsorbed molecules can desorb during the wait time, so we cannot do the same experiments at a temperature higher than 12-14 K probably. By reducing the temperature, we shall at least partly reduce the mobility of $\mathrm{D}_{2}$, except when it is already dominated by tunnel diffusion. We would maybe measure an increase in the NSC time at very low temperature.

EDwin Bergin: The o/p ratio of $\mathrm{NH}_{3}, \mathrm{CH}_{4}$ and $\mathrm{H}_{2} \mathrm{O}$ seen in comets are different but reflect a common spin temperature. If thermal evaporation determines the $\mathrm{o} / \mathrm{p}$ ratio, how could this be replicated?

Though we did not calculated specifically the case of $\mathrm{NH}_{3}$, I expect that it gives about the same kind of results than for water. The case of $\mathrm{CH}_{4}$ is different because the evaporation temperature range is rather different, there is less rotational constraints, so I don't know what could happen.

DAVID NEufELD: If I understood you correctly, the opr on the grain surface is always 3 , regardless of the formation temperature, but the para-water vaporizes slightly more 
rapidly, leading to an opr of 2.4. But what opr is expected when water ice are released by sputtering or photodesorption?

You understood perfectly! We did not considered sputtering or photodesorption processes, so I'd rather not answer. I can just say that if the sputtering eject randomly a unique molecule, the opr should be close to 3 , but if a small cluster of water ice is sputtered and thereafter it sublimates, then I expect more the value of 2.4 , but this is just my opinion.

E. F. van Dishoek: Just a follow-up on the question of David Neufeld. Photodesorption can change the opr from that in the ice. There are two basic mechanisms for $\mathrm{H}_{2} \mathrm{O}$ photodesorption (cf Andersson \& van Dishoek 2008, Arasa et al. 2010, 2011):

i) $\mathrm{H}_{2} \mathrm{O}$ dissociates in $\mathrm{H}+\mathrm{OH}$ and then recombines and escapes

ii) the energetic $\mathrm{H}$ resulting from photodissociation of $\mathrm{H}_{2} \mathrm{O}$ kicks out a neighboring water molecule.

In process i) the opr will change, in process ii) not. The relative importance of these processes varies with ice monolayer, but are both significant. 